\title{
Weak-Equivalence Principle Violation and Mass Change of Charged Matter due to Vacuum Polarization
}

\author{
M. Tajmar* \\ Institute of Aerospace Engineering, Technische Universität Dresden, Germany
}

(Dated: June 5, 2015)

\begin{abstract}
Vacuum polarization by electric fields is a well established fact. Assuming that anti-matter has negative gravitational properties, the fluctating electric dipoles from the quantum vacuum may also have gravitational dipolar properties. A model is developed that describes how electric fields could gravitationally polarize the vacuum causing gravitational screening or anti-screening effects. This leads to a violation of the Weak Equivalence Principle or a general mass change most notabily for elementary particles, such as the electron or positron, below but close to measured boundaries. Also a gravitational vacuum torque is predicted to act on a charged capacitor perpendicular to a gravitational field. The predictions could be verified by future laboratory experiments that could contribute on our understanding of the gravitational properties of anti-matter.
\end{abstract}

PACS numbers: 95.30.Sf, 42.50.Lc, 04.60.-m

\section{INTRODUCTION}

The universality of free-fall or the Weak Equivalence Principle (WEP) has been studied and verified since the time of Galileo Galilei. Almost all tests today have been carried out with neutral matter only due to the influence of the electromagnetic fields that can disturb the measurement. While such tests have achieved an Eötwash-factor sensitivity of $10^{-13}$ [1], only one test has been reported for the free-fall of an electron with its elementary charge at a sensitivity of only $10 \%$ [2]. Violations are currently expected from a quantum theory of gravity (see e.g. [3]) or from an extension to the standard model [4] but no theory for a WEP violation of charged matter has been proposed so far [5]. Recent work also suggests that electric fields [6] or rotation [7] may influence either mass itself or the gravitational attraction.

The influence of vacuum polarization on gravity has been studied in the 1980s by Long and others [8,9] who claimed that such polarization can lead to gravitational shielding. Recently, Hajdukovic [10-13] proposed a model showing that gravitational vacuum polarization can account for dark energy. His model involves the interaction of gravity with the virtual particles of the vacuum - a bridge that is presently not understood at all and frequently referred to as the cosmological constant problem (quantum theories predict a huge cosmological constant or space-time curvature that is not observed). Although speculative, Hajdukovic's work may shed some new light on that issue proposing an interesting solution as he predicts that anti-particles have negative gravitational masses. Here we propose to extend his model to take both electrical and gravitational polarization of the quantum vacuum into account. We show that this can lead to a WEP violation or a general mass change in particular for charged matter that can support the need for higher sensitivity tests of charged matter WEP tests.

*martin.tajmar@tu-dresden.de

\section{GRAVITATIONAL VACUUM POLARIZATION BY ELECTRIC FIELDS}

It is believed that the vacuum is filled with fluctuating particle and anti-particle pairs. For the electromagnetic case, the QED vacuum contains electron-positron pairs that can be polarized by external electric fields. This so-called vacuum polarization is well established and plays an important role in charge renormalization [14].

If anti-particles would have negative gravitational mass, also gravitational fields would polarize the vacuum. Recently, Hajdukovic [10-13] proposed that gravitational polarization of the QCD vacuum, that consists of virtual pion pairs, leads to a MOND-like behaviour and may serve as an alternative to the dark energy models in the universe. Blanchet et al [1518] also recently showed that MOND can be interpreted as a polarization of gravitational dipoles. Both assume that particles with negative gravitational mass have a positive inertial mass $\left(\overline{m_{g}}=-m_{g}, \overline{m_{i}}=m_{i}\right)$. This is a necessary requirement to also explain the behaviour of anti-matter particles that have been observed so far. In another recept paper, Villata [19] concludes that negative gravitational masses appear as a prediction of general relativity when CPT is applied.

We will extend the gravitational quantum vacuum model by considering that particle/anti-particle pairs can also have charge and therefore external electric fields should be able to gravitationally polarize the gravitational quantum vacuum as well. This includes charged virtual pions as well as electronpositron pairs. Since the electric field is much stronger than the gravitational field, such an electrically polarized vacuum can lead to much higher gravitational effects that may lead to experimental observation than gravitational polarization alone.

Since similar gravitational charges attract while similar electric charges repel, vacuum polarization with electric fields screen the source charge and polarization with gravitational fields anti-screen (amplify) the source mass. If we consider the QCD and QED vacuum, we see that negative electric charges should have a positive gravitational mass and positive electric charges a negative gravitational mass. For the case of the electron and positron, this assumption is clear. For 


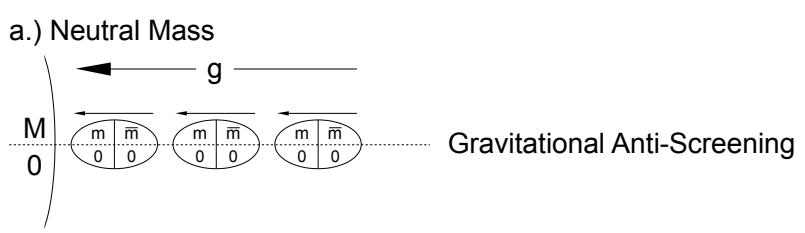

b.) Electrically Charged Mass

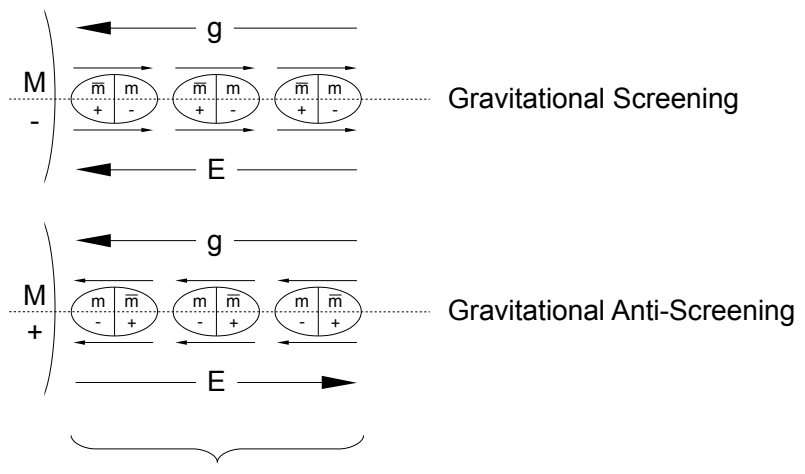

Virtual Particle-Dipoles

FIG. 1. Vacuum Polarization by Electric and Gravitational Fields

the charged pions we have to assume that quarks have a positive gravitational mass and anti-quarks a negative gravitational mass. By looking at the quark composition of the charged pions $\left(\pi^{+}=u \bar{d}, \pi^{-}=d \bar{u}\right.$ with $u=1.7-3.3 \mathrm{MeV} / \mathrm{c}^{2}$ and $d=4.1-5.8 \mathrm{MeV} / \mathrm{c}^{2}$ ), we come to the same conclusion as in the case of the electron-positron pair. Therefore, each virtual electric dipole is also associated with a virtual gravitational dipole.

By applying an electric field, we can now obtain a field from the vacuum that either screens or anti-screens the gravitational mass as illustrated in Fig. 1. A negative electric charge will screen and a positive electric charge will anti-screen the source mass. This is a very interesting behaviour that would obviously be of tremendous interest if it could be observed experimentally.

\section{A. Harmonic Oscillator Model}

In order to calculate the polarization effect, we will follow a similar approach as Leuchs et al [20], Urban et al [21] and Tajmar [22]. In the electric polarization case, where the attraction between electric charges dominates, the virtual dipole is modelled as a harmonic oscillator in the quasi-static limit. The distance $x_{0}$ between the dipoles is calculated as

$$
m x_{0} \omega_{0}^{2}=e E+\frac{Q}{|Q|} \cdot m g
$$

where $m$ and $e$ are the mass and charge of the virtual particle, $E$ and $g$ are the applied electric and gravitational field and $\omega_{0}$ is the fundamental resonance frequency associated with the quantum transition. It can be calculated from the virtual dipole rest mass as $\hbar \omega_{0}=2 m c^{2}$. The signum in Equ. 1 of the source charge $Q$ adds the gravitational contribution with the correct sign if the displacement $x_{0}$ is assumed always positive in our model.

We continue by calculating the gravitational dipole moment as $p_{g}=m x_{0}$. The gravitational polarization density is then given as

$$
P_{g}=N \cdot\left(\frac{e E}{\omega_{0}^{2}}+\frac{Q}{|Q|} \cdot \frac{m g}{\omega_{0}^{2}}\right)
$$

where $N$ is the density of virtual dipoles. It is usually assumed that this corresponds to the volume of the Compton wavelength of the virtual particle such that $N=1 / \lambda^{3}$ [20]. We will compute the associated vacuum mass density out of the polarization density using $\rho_{v}=-\nabla P_{g}$ and use the first Maxwell and Einstein-Maxwell equation [23, 24] for the divergence of the electric and gravitational field respectively. By integrating over the volume, the mass contribution from the vacuum polarization $M_{v}$ is given as

$$
M_{v}=\frac{e m}{16 \pi^{2} \epsilon_{0} h c} \cdot Q+\frac{Q}{|Q|} \cdot \frac{G m^{2}}{4 \pi h c} \cdot M
$$

where $M$ is the source mass and $\epsilon_{0}$ is the electric constant. Note that we have used the Compton wavelength and not the reduced Compton wavelength as in other approaches [10-12] for the virtual dipole density. Without charge, the vacuum contribution reduces to

$$
M_{v}=\frac{G m^{2}}{4 \pi h c} \cdot M
$$

Following Hajdukovic [10-12] and assuming in our case charged/neutral pions as the dominant vacuum contribution, we can calculate the case for neutral and charged sources as

$$
M_{v}=\left\{\begin{array}{cc}
1.5 \times 10^{-42} \cdot M & \text { for } \mathrm{Q}=0 \\
1.4 \times 10^{-13} \cdot Q+\frac{Q}{|Q|} \cdot 1.5 \times 10^{-42} \cdot M & \text { for } \mathrm{Q} \neq 0
\end{array}\right.
$$

It is interesting to note the small asymmetry between the electrical positive and negative case due to the additional gravitational polarization contribution.

\section{B. Weak Equivalence Principle Violation and Gravitational Vacuum Torque}

The effect may lead to two different observations:

1. Since the quantum vacuum influences the gravitational force, we may interpret this behavior as a violation of the weak equivalence principle. The gravitational mass would be affected while the inertial mass remains the same. 
2. If we follow Mach's principle, such that the inertial mass is due to the gravitational interaction with the rest of the universe, then the equivalence principle would still hold but we could observe a mass change depending on the body's charge.

The devitation in both cases (WEP violation or mass change) can be expressed by the factor $\eta$ as the ratio of the difference to the average of the affected and unaffected mass

$$
\eta=\frac{\left(M+M_{v}\right)-M}{\frac{\left(M+M_{v}\right)+M}{2}} \approx \frac{M_{v}}{M}
$$

For the case of the electron, the gravitational-electrical vacuum polarization correction becomes important. Equ. 5 predicts that the negative charge screens the electron's gravitational mass with $M_{v, \text { electron }}=-2.2 \times 10^{-32} \mathrm{~kg}$. For the positron, a gravitational mass increase is predicted by the same amount. This leads to a deviation of the Weak-Equivalence-Principle for both electrons and positrons at an order of $2.4 \%$. This is a factor of 5 below the only measurement by Witteborn et al [2] and should be detectable with modern means. For example, Dittus et al [25] proposed a space-based WEP test for positrons that should have a sensitivity level of $10^{-5}$. That could check the WEP violation possibility.

The second possibility, a general mass change of charged matter, is more difficult to observe as strong electric fields usually make mass measurements very difficult. However, a gravitational vacuum polarization effect may be detectable with strongly charged matter. For example, we will consider a plate capacitor with a high-k dielectric in between. The positive and negative charges will lead to a small positive and negative gravitational vacuum mass on each side, which will cause a torque in an ambient gravitational field due to vacuum polarization as

$$
\tau_{\mathbf{v}}=\mathbf{p}_{\mathbf{v}} \times \mathbf{g}=\left(M_{v} \cdot \mathbf{d}\right) \times \mathbf{g}
$$

where $d$ is the distance between the plates. Calculating the maximum case, when the gravitational dipole momentum is perpendicular to the ambient gravitational field as shown in Fig. 2, we can approximate the gravitational vacuum torque using Equ. 5 as

$$
\tau_{v, \max }=p_{v} \cdot g \approx 1.4 \times 10^{-13} \cdot Q \cdot d
$$

If we use $Q=C \cdot U$ and express the capacity as $C=\epsilon_{0} \cdot \epsilon_{r} \frac{A}{d}$, we can write the gravitational dipole momentum as

$$
\tau_{v, \max }=1.4 \times 10^{-13} \cdot \epsilon_{0} \epsilon_{r} \cdot A \cdot U
$$

where $\epsilon_{r}$ is the dielectric constant (or relative permittivity), $A$ the area of the capacitor and $U$ the charging voltage. Note that the final result does not depend on the dielectric thickness $d$ if we assume a plate thickness which is much smaller than the dielectricum's thickness. If we take values that are
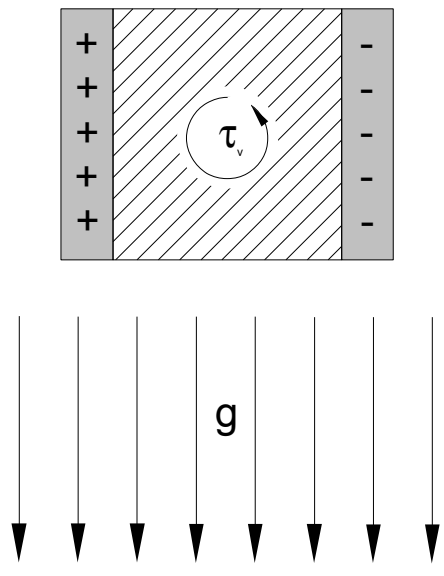

FIG. 2. Torque on Charged Capacitor

achievable in the laboratory such as $A=1 \mathrm{~m}^{2}, \epsilon_{r}=10,000$ $\left(=\mathrm{BaTiO}_{3}\right.$ at it's Curie point $\mathrm{T}=120{ }^{\circ} \mathrm{C}$ ) and $\mathrm{U}=100,000 \mathrm{~V}$, we get a maximum torque from the gravitational vacuum polarization of $\tau_{v, \max }=1.22 \times 10^{-14} \mathrm{Nm}$. Piezoresistive torque sensors have achieved a sensitivity in the range of $10^{-13} \mathrm{Nm}$ [26], which is just above the predicted gravitational vacuum torque from our capacitor example. Therefore, it does not seem unreasonable to test our model with experiments, provided that the capacitor is placed within a grounded cage in order to shield the electrostatic fields from the torque sensor (stray fields will cause major limitations in the precision of the experiment). It is also clear that the effect is so small that it could not have been detected in laboratory experiments without a dedicated setup.

In conclusion, we have developed a model that leads to an interesting coupling between electricity and gravitation through vacuum polarization, assuming that fluctuating electric dipole pairs in the quantum vacuum have also a gravitational dipole. This leads to a WEP violation or a general mass change most notably for elementary particles such as the electron or positron that is below present measurements bounds but should be accessible in future experiments. It also predicts a gravitational vacuum torque acting on a charged capacitor perpendicular to a gravitational field that may also be tested experimentally. Such tests may be more easily accessible than present efforts to measure the gravitational properties of anti-matter (e.g. [27-29]). 
[1] S. Schlamminger, K.-Y. Choi, T. A. Wagner, J. H. Gundlach, and E. G. Adelberger, Phys. Rev. Lett. 100, 041101 (2008).

[2] F. C. Witteborn and M. Fairbank, Phys. Rev. Lett. 19, 1049 (1967).

[3] A. Accioly and R. Paszko, Adv. Studies Theor. Phys. 3, 65 (2009).

[4] V. Kostelecký and J. Tasson, Phys. Rev. D 83, 016013 (2011).

[5] F. Rohrlich, Classical Charged Particles (Addison-Wesley, New York, 1965).

[6] M. Tajmar, J. Adv. Phys. 4, 219 (2015).

[7] M. Tajmar, J. Adv. Phys. , in Press (2015).

[8] D. Long, Nuovo Cimento 55B, 252 (1980).

[9] C. Shao-Guang, Nuovo Cimento 104B, 611 (1989).

[10] D. Hajdukovic, Astrophys. Space Sci. 330, 207 (2010).

[11] D. Hajdukovic, Europhys. Lett. 89, 49001 (2010).

[12] D. Hajdukovic, Astrophys. Space Sci. 334, 225 (2011).

[13] D. Hajdukovic, Mod. Phys. Lett. A 28, 1350124 (2013).

[14] B. Delamotte, Am. J. Phys. 72, 170184 (2004).

[15] L. Blanchet, Class. Quantum Grav. 24, 35293539 (2007).
[16] L. Blanchet, Class. Quantum Grav. 24, 35413570 (2007).

[17] L. Blanchet and A. LeTiec, Phys. Rev. D 78, 024031 (2008).

[18] L. Blanchet and A. LeTiec, Phys. Rev. D 80, 023524 (2009).

[19] M. Villata, Europhys. Lett. 94, 20001 (2011).

[20] G. Leuchs, A. Villar, and L.L.Sanchez-Soto, Appl. Phys. B 100, 9 (2010).

[21] M. Urban, F. Couchot, and X. Sarazin, "Does the speed of light depend upon the vacuum ?" (2011), arXiv:1106.3996v1, arXiv:1106.3996v1.

[22] M. Tajmar, Phys. Essays 25, 3 (2012).

[23] M. Tajmar and C. de Matos, J. Theoretics 3 (2001).

[24] C. Kiefer and C. Weber, Ann. Phys. 14, 253 (2005).

[25] H. Dittus and C. Lämmerzahl, Adv. Space Res. 39, 244 (2007).

[26] S. Kohout, J. Roos, and H. Keller, Rev. Sci. Instrum. 78, 013903 (2007).

[27] T. A. Collaboration and A. E. Charman, Nat. Comm. 4, 1 (2013).

[28] P. Scampoli and J. Storey, Mod. Phys. Lett. A 29, 1430017 (2014).

[29] P. I. et al, Hyperfine Interact. 228, 141 (2014). 